\title{
Nanopharmaceuticals and Their Applications in Bladder Cancer Therapy: a Mini Review
}

\author{
Nelson Durán*,a,b,c and Wagner J. Fávaro*,b,d \\ ${ }^{a}$ Laboratório de Química Biológica and \\ ${ }^{b}$ Laboratório de Síntese de Nanoestruturas e Interação com Biossistemas (NanoBioss), \\ Instituto de Química, Universidade Estadual de Campinas, 13083-970 Campinas-SP, Brazil \\ ${ }^{c}$ Núcleo de Nanomedicina (Nanomed), Universidade Federal do ABC (UFABC), \\ 09210-580 Santo André-SP, Brazil
}

${ }^{d}$ Laboratório de Carcinogênese Urogenital e Imunoterapia, Departamento de Biologia Estrutural e Funcional, Instituto de Biologia, Universidade Estadual de Campinas, 13083-970 Campinas-SP, Brazil

\begin{abstract}
The primary treatment for high-grade non-muscle invasive bladder cancer (NMIBC) is based on surgery by transurethral resection of bladder tumor (TURBT), followed by intravesical immunotherapy with Bacillus Calmette-Guerin (BCG) to prevent recurrence and to reduce the tumor progression. However, BCG therapy shows several undesirable effects. The current treatment on NMIBC is doxorubicin (DOX), but with high toxicity. Our nanotechnology strategy was done through scaffolds for the NMIBC treatment: graphene oxide (GO) and a nanostructured lipid carrier (NLC). A GO hybrid for administration of DOX and small interfering RNA (siRNA) was developed. This hybrids administered in vivo against NMIBC in rats gave absence of lesions. NLC was prepared by using a mixture of two lipids stabilized by a surfactant and DOX by high homogenization pressure technique. In this case showed a $20 \%$ of the animals exhibited benign lesions (papillary hyperplasia), however, in the presence of siRNA reached $40 \%$ of rats with benignant lesions. These two scaffolds are potential new drugs for DOX for bladder cancer treatment without any cardiotoxicity problems.
\end{abstract}

Keywords: cancer, bladder, graphene oxide, nanostructured lipid carrier, doxorubicin, siRNA

\section{Introduction}

\subsection{Bladder cancer}

Bladder cancer (BC) is the ninth most common type of cancer worldwide. ${ }^{1,2}$ In this year it was estimated that around 80,000 new cases would be diagnosed in the United States (ca. 60,000 and ca. 20,000 cases in men and women, respectively) with deaths in the US by $\mathrm{BC}$ may reach approximately 17,000 (12,000 men and ca. 5,000 women). ${ }^{3}$

Two of the most common types of $\mathrm{BC}$ are the non-muscle invasive bladder cancer (NMIBC) and muscle invasive bladder cancer (MIBC). The NMIBC is characterized by tumor formation in the urothelium (classified as Ta) (Figure 1). Lamina propria and muscles are not invaded by tumor cells. According to The European

*e-mail: duran@iqm.unicamp.br; wjfavaro@gmail.com This paper is part of the PubliSBQ Special Issue "IUPAC-2017" (http://publi.sbq.org.br/).
Association of Urology (EAU), tumors originating in the mucosa are classified as CIS (Tis). The CIS type tumors are superficial (flat shape); however, its diagnosis is uncertain, since CIS tumors are not detected by cystoscopy in many cases. Ta type corresponds to $70 \%$ of the cases and only $10 \%$ is related to CIS type in NMIBC. ${ }^{2}$

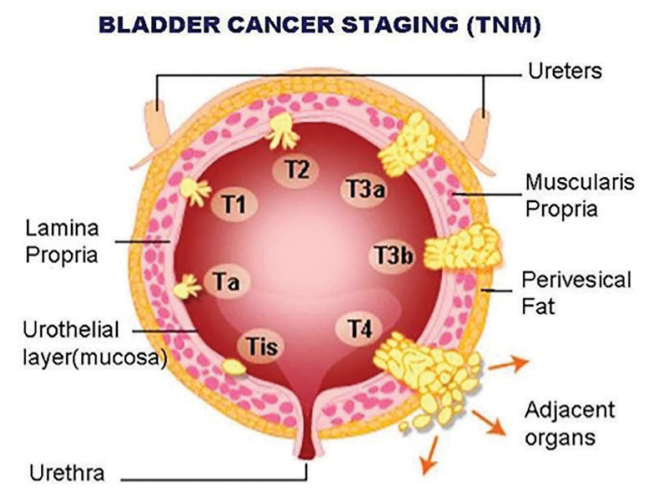

Figure 1. Bladder cancer staging. ${ }^{4}$ 


\subsection{Non-muscle invasive bladder cancer (NMIBC)}

The treatment of NMIBC represents a great challenge in the urology field because of the high recurrence and progression to the MIBC. ${ }^{5}$ It is known that intravesical administration of Bacillus Calmette-Guérin (BCG) is effective (i.e., decreasing the recurrence rate), but approximately $40 \%$ of patients undergoing BCG treatment may show progression to the invasive form of $\mathrm{BC} .^{2}$ As a consequence, it is of great importance to develop innovative drug delivery systems (i.e., nanostructured) which allow intravesical drug administration better than the usual drugs. Besides these facts, these delivery platforms should enhance drug absorption by the bladder tissues in order to trigger an efficient therapeutic response, avoiding the progression of NMIBC to MIBC. ${ }^{5-7}$ It is important that the intravesical drug administration consists on the reduced side effects compared to systemic administration and also to the higher drug bioavailability.

\subsection{Muscle invasive bladder cancer (MIBC)}

At the stage of T2 up to T4 radical cystectomy is regarded as the standard approach to the treatment of invasive bladder cancer in the United States. This involves the removal of regional pelvic lymph nodes followed by an en bloc resection of the bladder, prostate and seminal vesicles in men or, in women, an anterior exenteration in which the bladder is removed along with the ovaries, uterus, urethra, and a segment of the anterior wall of the vagina. It is important to note the benefit of an extensive and thorough lymphadenectomy. However, in this stage, the chemo- or immunotherapy are difficult to apply.

\subsection{Nanoscaffolds for NMIBC}

In our laboratories the strategy to use the nanotechnology was to create two main scaffolds for the NMIBC treatment: graphene oxide and nanostructured lipid carriers to administrate doxorubicin (DOX) in rats, as one of the most important drug on these treatments (Figure 2).

The second scaffold, that is the nanostructured lipid carrier was prepared by using a mixture of two lipids, a solid (butter from Amazonian fruit) with a liquid lipid (oil from Amazonian fruit) stabilized by a mixture of surfactants, a tribloc polymer of ionic nature and/or with cationic natures incorporating DOX by the high homogenization pressure technique.

\subsection{Graphene oxide as scaffold}

The graphene (GO) sample: thickness 0.7-1.2 nm (atomic force microscopy, AFM); ca. 300-800 nm (X and Y dimensions) the standard size $<450 \mathrm{~nm}$ and 1-20 $\mu \mathrm{m}$ lateral dimensions from Cheap Tubes Inc., Bratleboro, USA was selected for our study, single-layer graphene oxide, purity $99 \%$. Exhaustive characterization of GO was afforded by spectroscopic and microscopic techniques (Figure 3).

This characterization is of extremely importance, since it is absolutely necessary to know exactly the characteristic of any nanostructure before any biological study.
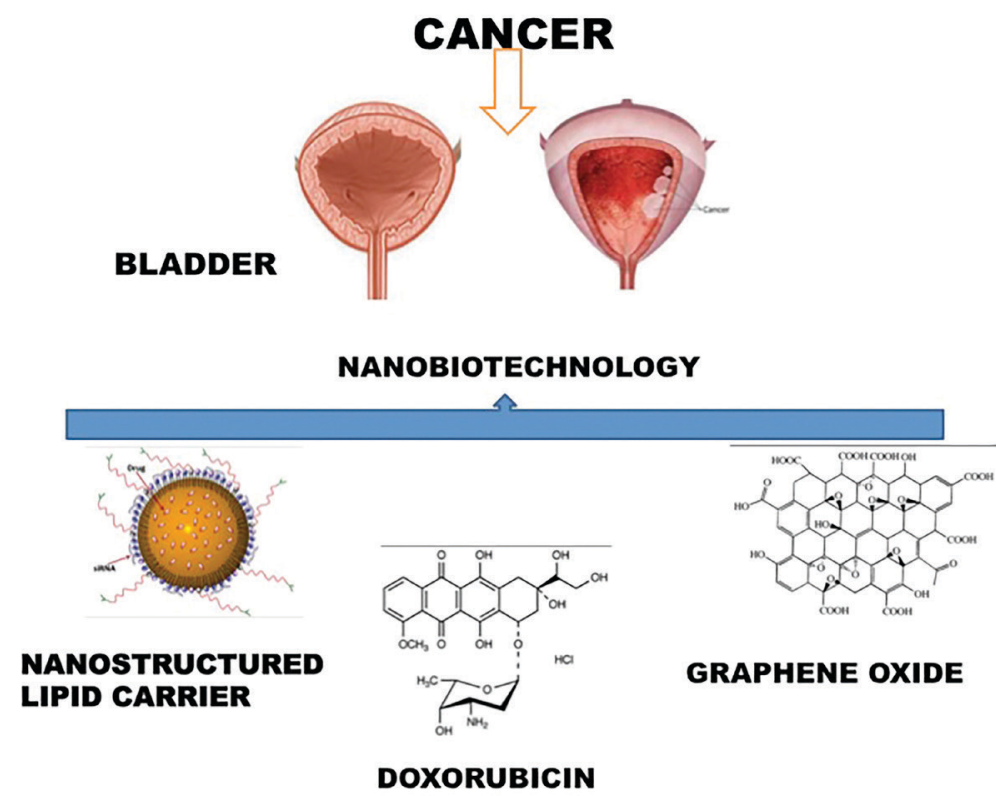

Figure 2. Nanoscaffolds as strategy to treat NMIBC in which we have different scaffols for bladder cancer, such as, nanostructured lipid carriers, free doxorubicin and graphene oxide. 


\section{CHARACTERIZATION OF GRAPHENE OXIDE}

Source: Single layer, $99 \%$ purity (average thickness $0.7-1.2 \mathrm{~nm} ; \sim 300-800 \mathrm{~nm}$ X\&Y dimensions standard size<450nm \& 1-20um lateral dimensions) comercial (Cheap Tubes Inc., Bratleboro, USA (batch data: 6/26/2013)

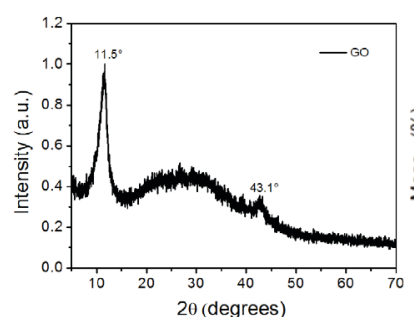

X-Difractometer Shimadzu, model XRD7000, under radiation CuKa $(\lambda=1,54060 \AA)$, at $40 \mathrm{kV}$ current $30 \mathrm{~mA}$, scanning at $2^{\circ} \mathrm{min}^{-1}$.

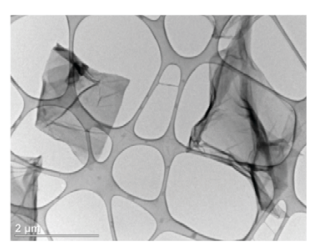

TEM

TEM-MSC $2100-J E O L$

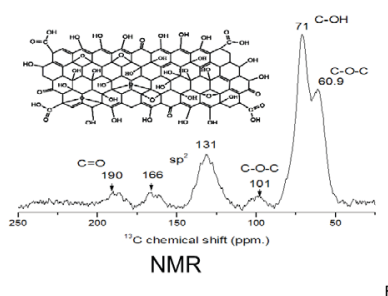

NMR- ${ }^{13}$ C-Bruker 300 , at $300 \mathrm{MHz}$

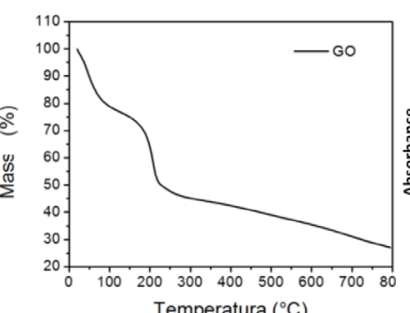

Temperatura $\left({ }^{\circ} \mathrm{C}\right)$

Thermogravimetric analyzer TA-STD- Q600 , heating rate of $10^{\circ} \mathrm{C} \mathrm{min}$ $^{-1}$ and gas flow of $100 \mathrm{~mL} \mathrm{~min}^{-1}$ nitrogen.

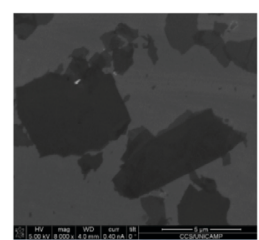

FEG-SEM

NanoLab200-FEI

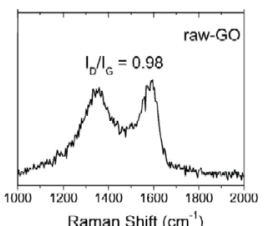

Raman

Raman Scattering Spectroscopy (Witec) with excitation by Art-ion laser $(l=633 \mathrm{~nm})$

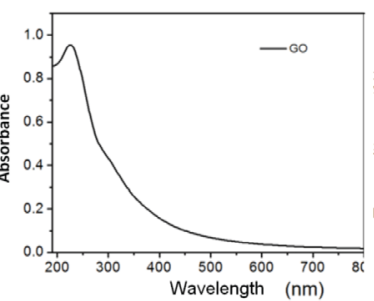

Spectrophotometer UV-vis Zhimadzu, UV-1650PC.

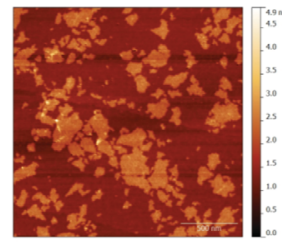

AFM

AFM-Nanoscope III - Digital Instruments

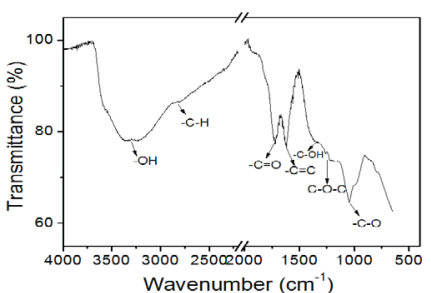

Wavenumber $\left(\mathrm{cm}^{-1}\right)$

Spectrophotometer Agilent Technol. Cary 630 FTIRmode ATR). Resolution $4 \mathrm{~cm}^{-1}$

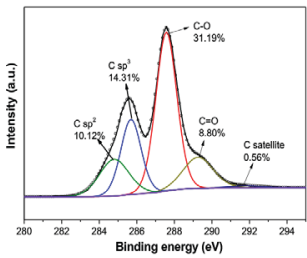

XPS (C1s)

K-Alpha X-ray Photoelectron Spectrometer (XPS) Thermo.
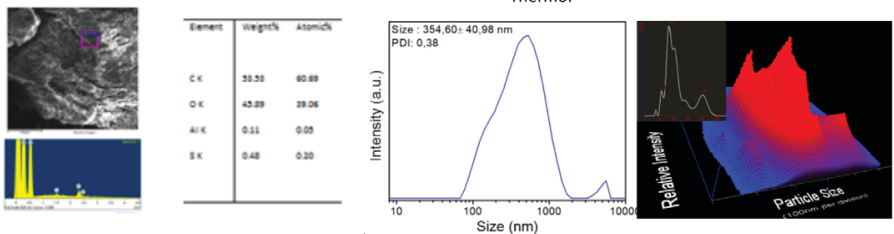

EDS-FESEM

The analysis of Energy-dispersive X-ray spectroscopy (EDS) (Field Emission Scannin
(Inspect equipment FEI-F50)

NTA

NanoSight LM-10

Figure 3. Total characterization of the commercial graphene oxide (partially presented at reference 8).

Figure 3 shows the typical graphene oxide structure from several techniques, and the results are in agreement with data from the literature.

\subsection{Synthesis of the graphene oxide derivatives}

The coupling of small interfering RNA (siRNA) to GO (gaphene oxide))-PEG (6 ARM-poly(ethylene glycol) amine)-PEI (polyethyleneimine) was important, since oncogene overexpression is one of the major causes of urothelial carcinoma; therefore, the silencing of oncogenes via siRNA coated with a platform may provide an effective approach to the prevention of bladder cancer (Figure 4).

The reaction was performed according to methods described in literature, with some modifications. ${ }^{9}$ Briefly: a sample of $10 \mathrm{mg}$ of GO (carboxylated) ${ }^{10}$ was dispersed in deionized water and $10 \mathrm{mg}$ of previously dispersed six-armed PEG (PEG (6 ARM-poly(ethylene glycol) amine or 6 ARM-PEG-amine) $(15 \mathrm{kD})$ in the deionized water was added to the GO dispersion and kept in an ultrasonic bath for $5 \mathrm{~min}$. Ten $\mathrm{mg}$ of hydrochloride 1-ethyl3-(3-dimethylaminopropyl)carbodiimide (EDC) dissolved in deionized water was added to the mixture, with stirring for $15 \mathrm{~min}$. After this, $50 \mathrm{mg}$ of PEI dispersed in deionized water was added and kept in the ultrasonic bath for $5 \mathrm{~min}$. Finally, an additional $20 \mathrm{mg}$ of EDC dissolved in deionized water was added. The mixture was kept under constant stirring for $6 \mathrm{~h}$. Centrifuged and resuspension in deionized water to remove unreacted PEG and PEI with GO. Then, it was added siRNA as previously reported. ${ }^{11}$ The oxidized $\mathrm{GO}$ and doxorubicin was prepared as also an intermediate following the procedure previously reported. ${ }^{10}$

Attenuated total reflectance (ATR), X-ray diffraction analysis (XRD) and X-ray excited photoelectron spectroscopy (XPS) were used for characterization, besides other usual techniques. ${ }^{10}$ The size and zeta potential of this nanoparticles were $149.0 \pm 10.0 \mathrm{~nm}$ and $+37.2 \pm 0.6 \mathrm{mV}$, respectively. All the intermediates were also analyzed and described on Table 1.

Figure 5 shows the synthesis of graphene oxide and doxorubicin (DOX) by reaction in the presence of EDC (hydrochloride 1-ethyl-3-(3-dimethylaminopropyl) carbodiimide)/NHS ( $N$-hydroxysuccinimide). This is another functionalized GO after oxidizing the surface where 

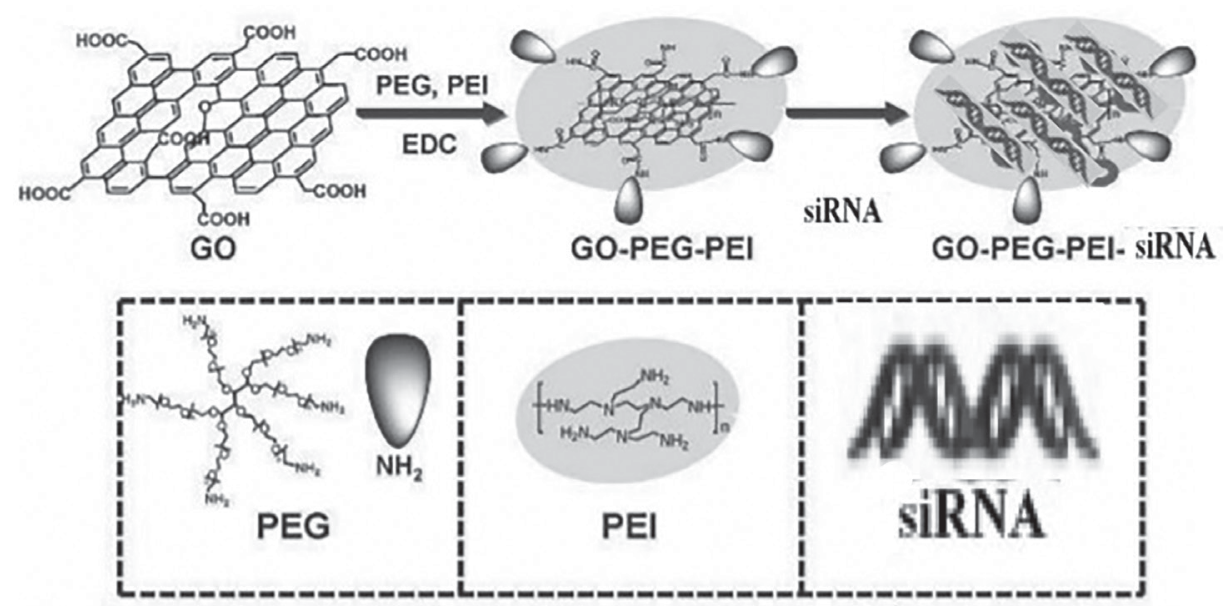

Figure 4. Synthesis of gaphene oxide (GO)-PEG (6 ARM-poly(ethylene glycol) amine or 6 ARM-PEG-amine) (PEG)-polyethyleneimine (PEI)-siRNA (adapted from reference 11).

Table 1. Particle size and zeta potential of GO, GO-PEG-PEI, GO-COOH and GO-COOH-DOX

\begin{tabular}{lcc}
\hline Sample & Particle size / $\mathrm{nm}$ & Zeta potential / $\mathrm{mV}$ \\
\hline GO & $133 \pm 41$ & $-31.2 \pm 7.9$ \\
GO-PEG-PEI & $149 \pm 10$ & $+37.2 \pm 0.6$ \\
GO-COOH & $126 \pm 29$ & $-27.9 \pm 4.4$ \\
GO-COOH-DOX & $153 \pm 10$ & $-36.2 \pm 0.6$ \\
\hline
\end{tabular}

GO: graphene oxide; PEG: 6 ARM-poly(ethylene glycol) amine or 6 ARM-PEG-amine; PEI: polyethyleneimine; DOX: doxorubicin.

were formed carboxylated groups and covalently bound DOX.

The method followed procedures reported in literature, ${ }^{12,13}$ with some modifications in the presence of $\mathrm{NaOH}$ and chloroacetic acid. The intermediates in the synthesis of GO-PEG-PEI are shown in the Table 1 and the final product was $153 \pm 10 \mathrm{~nm}$ and zeta potential of $-36.2 \pm 0.9 \mathrm{mV}$ (Figure 5). ${ }^{10}$

\section{Cytotoxicity}

\subsection{Hemolysis}

The hemolytic assay was carried out following the standard procedure from ASTM E2524-08 (standard test method for analysis of hemolytic properties of nanoparticles), as applied by Durán $e t$ al. ${ }^{8}$ Hemolytic activity assay was also performed according to our earlier report. ${ }^{14}$ The hemolytic assay following the procedure above showed completely absence of hemolysis by graphene oxide up to $100 \mu \mathrm{g} \mathrm{mL}^{-1}$.

\subsection{Cell viability}

In order to verify the toxicity of DOX, DOX-COOH and GO-COOH-DOX, cytotoxicity studies on epithelial cells A5637 (bladder cancer cells) $\left(1.5 \times 10^{4}\right.$ cells per well verified by 3-(4,5-dimethyl-2-thiazolyl)-2,5-diphenyl$2 \mathrm{H}$-tetrazolium brimide (MTT) assay after $24 \mathrm{~h}$ ) were

\section{DOXORUBICIN(DOX)}

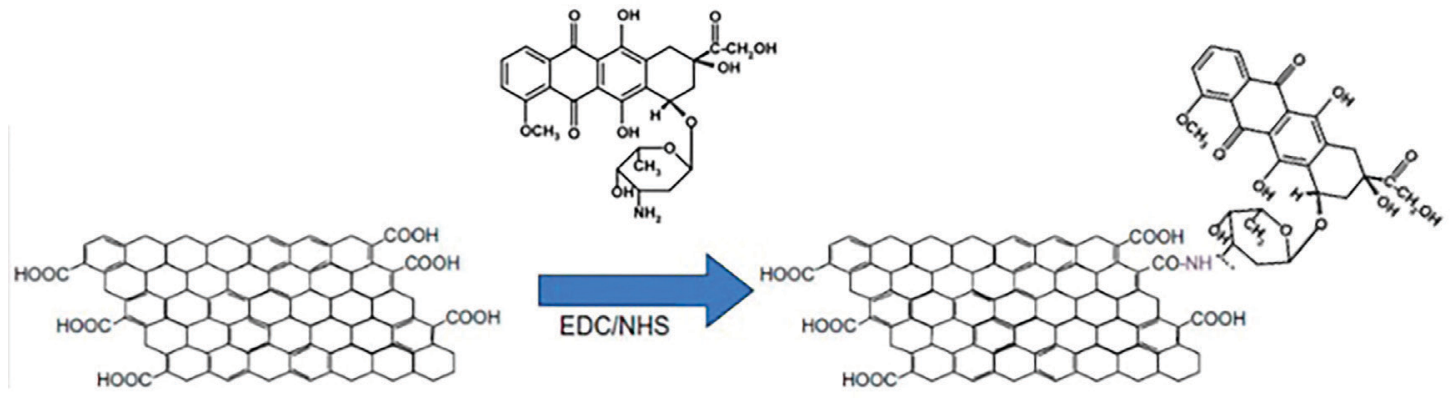

\section{GO-COOH}

Figure 5. Synthesis of functionalized GO with DOX I the presence of EDC/NHS. ${ }^{10}$ 
performed. DOX showed an accentuated toxicity against bladder cancer tumor cells. The half maximal inhibitory concentration $\left(\mathrm{IC}_{50}\right.$ ) value of DOX was around $6 \mu \mathrm{gL}^{-1}$. Previously, GO cytotoxicity was found that up to $50 \mu \mathrm{g} \mathrm{mL}^{-1}$ exert no toxicity to 14 cell cultures and to lymphocytes and some of them up to $100 \mu \mathrm{gL}^{-1} \cdot{ }^{6} \mathrm{GO}-\mathrm{COOH}$ did not reach the $\mathrm{IC}_{50}$ value. However, GO-COOH-DOX system showed an $\mathrm{IC}_{50}$ of about $10 \mu \mathrm{g} \mathrm{mL}^{-1}$. The $\mathrm{X}$ axis of the graph represents the concentration of the total amount of the nanoparticles, and not just the relative DOX concentration. Considering the DOX concentration added initially in the nanoparticles the $\mathrm{IC}_{50}$ of the drug when incorporated into GO-COOH-DOX was about $3.33 \mu \mathrm{g} \mathrm{mL}^{-1}$. Therefore, GO-COOH was able to promote an increase in the toxicity of DOX compared to the drug in the free form and could play a satisfactory effect considering the in vivo application for the treatment of NMIBC. ${ }^{10}$

Econanotoxicology of GO exhibited nontoxic effect in the in vitro or in vivo assay with the standard organisms, excepting with Hydra for $96 \mathrm{~h}$ of contact. ${ }^{8}$

\subsection{In vivo toxicity}

The systemic toxicity was correlated with the degree of inflammation in organs: the peritoneum, liver and spleen of graphene oxide. Evaluation of the systemic toxicity of GO solutions showed signs of inflammation only in the spleen. Deposition on the peritoneum was observed. The degree of inflammation was assessed by a semi-quantitative scale.

\section{Systemic Toxicity in vivo}

\subsection{Histophatological analysis}

Inflammation in the spleen ad peritoneum and no effect on liver were observed at $5 \mu \mathrm{g} \mathrm{g}^{-1}$ (1 $\mathrm{mg}$ per rat). In lower concentration very slight inflammation in all the organs was observed.

\subsection{Genotoxicity (micronucleous) and biochemical analysis}

The results showed that serum levels of glutamate pyruvate transaminase (GPT), glutamate oxalacetate transaminase (GOT), urea and creatinine in treated animals $24 \mathrm{~h}$ with GO $0.05 \mu \mathrm{g} \mathrm{g}^{-1}$, GO $0.5 \mu \mathrm{g} \mathrm{g}^{-1}$ and GO $5.0 \mu \mathrm{g} \mathrm{g}^{-1}$ did not differ significantly between them, as well as the control group. Similar results with the other GO derivatives were found (unpublished results).

\subsection{In vivo experiments on rat's bladder cancer}

For this experiment 35 rats of the Fisher 344 strain, aged 7 weeks, weighing on average 170 grams, were obtained from the Bioethics Center of the State University of Campinas (CEMIB/UNICAMP) following the ethical principles in animal research (Protocol CEUA 3795-1). There was a standardized induction procedure for NMIBC with the use $N$-methyl- $N$-nitrosourea (MNU). ${ }^{15}$ The protocol of the bladder cancer induction (NMIBC) and treatment is described in Figure 6.

> METHODOLOGY: BLADDER CANCER INDUCTION: INTRAVESICULAR

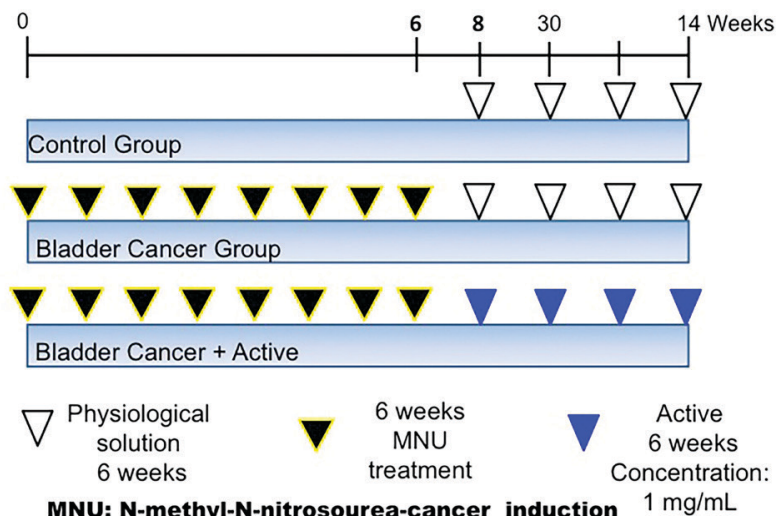

Figure 6. The protocol followed in the induction of NMIBC and the treatment of rats with nanostructures: control group, bladder cancer group and bladder cancer by the active treatment.

According to the Table 2, for the group treated with GO-PEG-PEI/siRNA, $60 \%$ of the animals showed malignant lesions classified as carcinoma in situ (pTis), $20 \%$ of the animals had normal bladder and $20 \%$ exhibited low grade $\mathrm{pTa}$.

Based on the results, GO-PEG-PEI/siRNA system was able to reduce the aggressiveness of the NMIBC. In the DOX group $100 \%$ of the animals presented lesions classified as high grade $\mathrm{pTa}$, a diagnosis even worse than that presented by the MNU (cancer) group. A possible explanation would be the high solubility of the drug in water, resulting in rapid elimination of the drug from the urine. In addition, a low drug permeability in the urothelium may reduce drug efficacy when administered intravesically.

Considering the use of DOX for the treatment of NMIBC, previous studies with a commercial formulation (Doxil $®$ ), administered intraperitoneally at a dose of $3.0 \mathrm{mg}$ per $\mathrm{kg}$, revealed that $20 \%$ of the animals showed benign lesions and $80 \%$ in situ papillary carcinoma malignant lesions. ${ }^{16} \mathrm{~A}$ drug against Cisplatin tumors refractory to BCG immunotherapy, the Valrubicin, a semisynthetic analogue of DOX showed effectiveness in less than $10 \%$ of treated patients in two years and ineffectiveness in $\mathrm{T} 1$ stage cases. ${ }^{17}$

As for animals treated with GO-COOH-DOX, 20\% of the animals presented normal bladder, $20 \%$ pTis-like 
Table 2. Histopathology and tumor staging of different experimental groups $(n=5)$

\begin{tabular}{|c|c|c|c|c|c|c|c|c|}
\hline Histopathology & Control & $\begin{array}{c}\text { MNU } \\
\text { (Cancer) }\end{array}$ & GO-PEG-PEI & $\begin{array}{l}\text { GO-PEG-PEI/ } \\
\text { siRNA }\end{array}$ & DOX & GO-COOH-DOX & $\begin{array}{c}\text { GO-PEG-PEI/ } \\
\text { siRNA + } \\
\text { GO-COOH-DOX }\end{array}$ & Lesion \\
\hline Normal & $5(100)$ & - & - & $1(20)$ & - & $1(20)$ & $3(60)$ & - \\
\hline Papillary hyperplasia & - & - & - & - & - & - & - & benign \\
\hline Low-grade intraurothelial neoplasia & - & - & - & - & - & - & - & pre-malignant \\
\hline Flat carcinoma in situ (pTis) & - & $1(20)$ & $4(80)$ & $3(60)$ & - & $1(20)$ & $1(20)$ & malignant \\
\hline $\begin{array}{l}\text { Low-grade papillary carcinoma } \\
\text { (pTa) }\end{array}$ & - & $1(20)$ & - & $1(20)$ & - & - & - & malignant \\
\hline $\begin{array}{l}\text { High-grade papillary carcinoma } \\
\text { (pTa) }\end{array}$ & - & $3(60)$ & - & - & $5(100)$ & $3(60)$ & $1(20)$ & malignant \\
\hline $\begin{array}{l}\text { Tumor invading lamina propria } \\
\text { (pT1) }\end{array}$ & - & - & $1(20)$ & - & - & - & - & malignant \\
\hline $\begin{array}{l}\text { Squamous metaplasia associated } \\
\text { to pT1 }\end{array}$ & - & - & - & - & - & - & - & malignant \\
\hline
\end{tabular}

MNU: $N$-methyl- $N$-nitrosourea; GO: graphene oxide; PEG: 6 ARM-poly(ethylene glycol) amine or 6 ARM-PEG-amine; PEI: polyethyleneimine; DOX: doxorubicin; siRNA: small interfering RNA; values in parentheses show the percentage.

lesions and $60 \%$ high-grade pTa. Thus, it may be suggested that the GO-COOH-DOX system had a promising effect by promoting the reduction of NIMBC aggressiveness, although with aggressive tumors in some animals. Finally, $60 \%$ of the animals exhibited a normal bladder diagnosis for the association between the GO-COOH-DOX and GO-PEG-PEI/siRNA. For the same group, $20 \%$ of the animals had pTis lesions and the remaining $20 \%$ presented high grade pTa associated with squamous metaplasia. Considering these results, it can be inferred that the association between GO-COOH-DOXO and GO-PEG-PEI/siRNA revealed great potential for the treatment of NIMBC. ${ }^{11}$

\subsection{Nanostructured lipid carrier as scaffold}

The nanostructured lipid carrier (NLC) were prepared following the previous reports of our laboratories (Figure 7). ${ }^{18-21}$ Briefly: first, the two solid lipids in the group comprising Cupuaçu butter and bis-diglyceryl polyacyladipate- 2 were mixed in a mass ratio of 70:30\% to melt at a temperature in the range of about $70{ }^{\circ} \mathrm{C}$. Soon the liquid lipid oil of Buriti in the range around $20 \%$, to the solid lipids Doxorubicina, in the range of $5 \%$ to the lipid composition remained under agitation until the complete dispersion of the drug. Next, the composition obtained was added to an aqueous solution containing a mixture of surfactants, in the range of about 50:50\% (Pluronic), with behentrimonium chloride at a concentration of $0.8 \%$, by the ratio of total solution, previously prepared separately and at a temperature at $70{ }^{\circ} \mathrm{C}$; under mechanical stirring in UltraTurrax at a rotation in the range of $5000 \mathrm{rpm}$, for about
30 min forming a nanoparticle emulsion. The obtained nanocarriers have a size in the range of 170-220 nm, positively charged surface with zeta potential in the range of +50.0 to $+80.0 \mathrm{mV}$ and stability time of about 50 days. The siRNA was added as reported above. ${ }^{10}$

\subsection{Cytotoxicity}

The cytotoxicity of NLC with and without DOX was carried out by MTT assay on epithelial cells A5637. $\mathrm{IC}_{50}$ values for NLC, DOX and NLC-DOX were 50, 6 and $3 \mu \mathrm{g} \mathrm{mL} \mathrm{m}^{-1}$, respectively. Then, the encapsulated DOX in NLC was more effective against these cells.

\subsection{In vivo toxicology}

Toxicology studies of NLC on rats showed very little or almost nonexistent toxicity for various routes, such as oral, dermal or intravenous administration, in a similar way as in the literature. ${ }^{22}$

\subsection{In vivo experiment on rats with bladder cancer with nanostructured lipid carrier}

Regarding the use of doxorubicin for the treatment of NMIBC, previous studies with a commercial formulation (Doxil@), using a dose of $3.0 \mathrm{mg} \mathrm{kg}^{-1}$ equivalent administered intraperitoneally, demonstrated that $20 \%$ of the animals had benign lesions and $80 \%$ malignant lesions of the papillary carcinoma type in situ. ${ }^{16}$ The results with the use of NLC-DOX developed in our laboratory showed that $20 \%$ of the animals presented benign lesions 


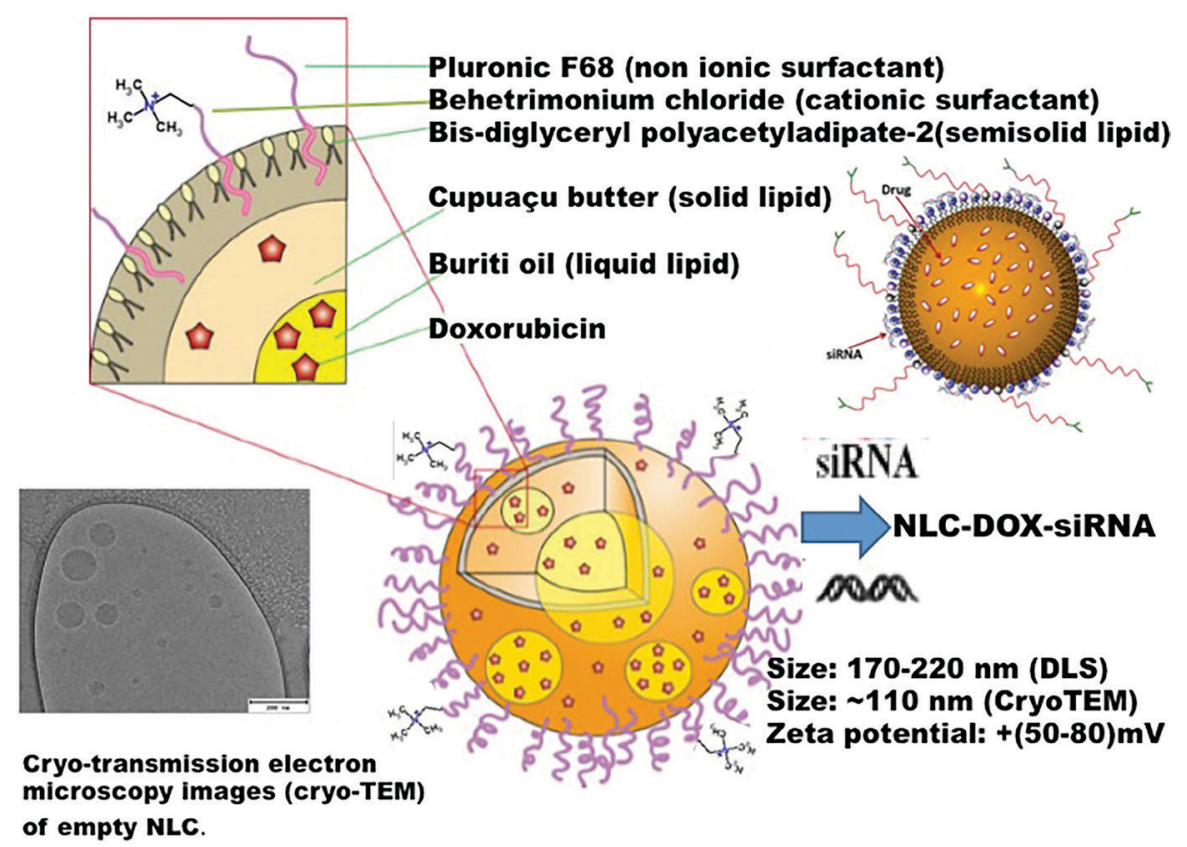

Figure 7. Scheme of preparation and characterization of NLC loading with DOX (adapted from reference 23). This scheme represents all the procedures followed for the synthesis of NLC and DOX.

(papillary hyperplasia) and 80\% malignant lesions (Table 3). On the other hand, considering the malignant lesions, $40 \%$ of them were papillary high grade (pTa), $20 \%$ papillary low grade (pTa) and 20\% urothelial carcinoma invading lamina propria (pT1). It is worth mentioning that in the case of refractory CIS tumors, Valrubicina, which is a semi-synthetic analogue of DOX is the medicine approved by the Food and Drug Administration (FDA, USA) for the treatment of this disease. On the other hand, this treatment is effective in less than $10 \%$ of patients treated in 2 years. ${ }^{17}$

Then, these platforms showed to be quite efficient in the bladder cancer. Summarizing the results, the data shown that in the case of NMIBC histopathological observation was observed a $60 \%$ of recovery to normal stage in the presence of NGO-PEO-PEI/siRNA-
NGO-DOX and $40 \%$ of normal for NLC-DOX/siRNA treatments. This was more efficient than the DOX alone in which was exhibited only $25 \%$ of benign papillary hyperplasia (Table 3). Besides this low efficiency DOX is also associated to cardiotoxicity.

\section{Final Remarks}

Finally, both scaffolds GO or NLC associated to DOX were effective on the bladder cancer on rats exhibiting NMIBC. Up to $60 \%$ of the animals exhibited a normal bladder diagnosis for the association between the GO-COOH-DOX and GO-PEG-PEI/siRNA. Considering these results, it can be inferred that the association between GO-COOH-DOX and GO-PEG-PEI/siRNA revealed great potential for the treatment of NMIBC.

Table 3. Histopathology and tumor staging of different experimental groups $(n=5)$

\begin{tabular}{|c|c|c|c|c|c|c|c|c|}
\hline Histopathology & Control & $\begin{array}{c}\text { MNU } \\
\text { (Cancer) }\end{array}$ & DOXO & NLC & NCL-DOX & $\begin{array}{l}\text { NCL-DOX/ } \\
\text { siRNA }\end{array}$ & BCG & Lesion \\
\hline Normal & $5(100)$ & - & - & - & - & - & - & - \\
\hline Papillary hyperplasia & - & - & $1(25)$ & - & $1(20)$ & $2(40)$ & - & benign \\
\hline Low-grade intraurothelial neoplasia & - & - & - & - & - & - & - & pre-malignant \\
\hline Flat carcinoma in situ (pTis) & - & - & - & - & - & $1(20)$ & - & malignant \\
\hline Low-grade papillary carcinoma (pTa) & - & $1(20)$ & - & $1(20)$ & $1(20)$ & $2(40)$ & - & malignant \\
\hline High-grade papillary carcinoma (pTa) & - & $2(40)$ & $2(50)$ & $2(40)$ & $2(40)$ & - & - & malignant \\
\hline Tumor invading lamina propria (pT1) & - & $2(40)$ & $1(25)$ & $1(20)$ & $1(20)$ & - & $2(40)$ & malignant \\
\hline Squamous metaplasia associated to $\mathrm{pT} 1$ & - & - & - & $1(20)$ & - & - & $3(60)$ & malignant \\
\hline
\end{tabular}

MNU: $N$-methyl- $N$-nitrosourea; DOX: doxorubicin; NLC: nanostructured lipid carrier; siRNA: small interfering RNA; BCG: Bacillus Calmette-Guerin; values in parentheses show the percentage. 
In other hand, the results with the use of NLC-DOX-siRNA showed that $40 \%$ of the animals presented normal recovered. This probably will be enhanced by different protocol that it was used in these experiments. Then, we expect that, in this case, we could get better results in other protocol. Important is that both scaffold notoriously diminish the cardiotoxicity of DOX.

\section{Acknowledgments}

Support from NanoBioss (MCTI), INOMAT (CNPq/ MCTI), CNPq and FAPESP are acknowledged.

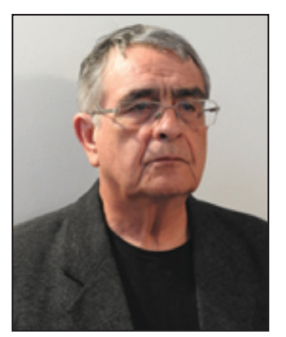

Nelson Durán is a Professor of Chemistry at the Universidade Estadual de Campinas, UNICAMP (Brazil). He received his $P h D$ at University of Porto Rico (USA). Associated Professor at the Universidad Católica de Valparaiso, Chile and carried out Visiting Professorship at Universidade de São Paulo, Brazil. In 1978, he joined the Chemistry Institute of UNICAMP (Brazil) working in Biological Chemistry and Biotechnology. His present research interests are nanobiotechnology in cosmetics and in pharmaceuticals, besides metallic nanoparticles as antibiotics and anticancer carriers, and in carbon and silica nanocarriers. He is the Coordinator of the Brazilian Nanotoxicology Network; Member of INOMAT (MCTI/CNPq) and Vice Coordinator of NanoBioss (MCTI) and Member of Brazilian-NanoReg-European Community, in vivo nanotoxicology. He published 444 papers (ISI) in chemistry, biochemistry, biotechnology and in nanobiotechnology and four Books in the Nanotechnology area. He registered 65 patents.

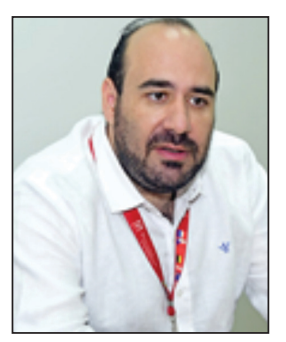

Wagner José Fávaro is a Professor of Anatomy, Pathology and Oncology at the University of Campinas, UNICAMP (Brazil). He received his Master (2006), $\mathrm{PhD}$ (2009) and Post-doctorate (2011) at UNICAMP. Since 2012, he is Assistant Professor at Department of Structural and Functional Biology and Coordinator of Urogenital Carcinogenesis and Immunotherapy Laboratory (LCURGIM) at UNICAMP. His present research interests are uropathology, carcinogenesis, urogenital tumors, immunotherapy, regenerative medicine and nanobiotechnology in oncology. Responsible for the development of the OncoTherad immunomodulator, which has therapeutic application in several tumors. He is a Member of the NANoREG ( a common European approach to the regulatory testing of Manufactured Nanomaterials).

\section{References}

1. Witjes, J. A.; Lebret, T.; Comperat, E. M.; Cowan, N. C.; de Santis, M.; Bruins, H. M.; Virginia Hernandez, V.; Espinos, E. L.; Dunn, J.; Rouanne, M.; Neuzillet, Y.; Veskimae, E.; van der Heijden, A. G.; Gakis, G.; Ribal, M. J.; Eur. Urol. 2017, 71, 462.

2. Babjuk, M.; Bohle, A.; Burger, M.; Capoun, O.; Cohen, D.; Comperat, E. M.; Hernandez, V.; Kaasinen, E.; Palou, J.; Roupret, M.; van Rhijn, B. W. G.; Shariat, S. F.; Soukup, V.; Sylvester, R. J.; Richard Zigeune, R.; J. Urol. 2017, 71, 447.

3. Siegel, R. L.; Miller, K. D.; Jemel, A.; Ca-Cancer J. Clin. 2017, 67,7 .

4. http://diseasesdoctor.com/bladder-cancer-symptoms-causessigns-treatment, assessed on December 25, 2017.

5. Cavaliere, C.; D’Aniello, C.; Cecere, S. C.; di Napoli, M.; Berretta, M.; Franco, R.; Pisano, C.; Perdonà, S.; Setola, S.; Fisichella, R.; Spartà, D.; Tambaro, R.; Pignata, S.; Facchini, G.; World J. Cancer Res. 2014, 1, e126.

6. Martin, D. T.; Hoimes, C. J.; Kaimakliotis, H. Z.; Cheng, C. J.; Zhang, K.; Liu, J.; Wheeler, M. A.; Kelly, W. K.; Tew, G. N.; Saltzman, W. M.; Weiss, R. M.; Nanomedicine: NBM 2013, 9 , 1124.

7. Gilligan, T. D.; Steele, G. S.; Zietman, A. L.; Kantoff, P. W. In Holland-Frei Cancer Medicine, $6^{\text {th }}$ ed.; Kufe, D. W.; Pollock, R. E.; Weichselbaum, R. R.; Bast Jr., R. C.; Gansler, T. S.; Holland, J. F.; Frei III, E., eds.; BC Decker Inc.: Hamilton, Canada, 2003, ch. 10 .

8. Durán, N.; Martinez, D. S. T.; Justo, G. Z.; de Lima, R.; de Castro, V. L.; Umbuzeiro, G. A.; Barbieri, E.; Durán, M.; Melo, P. S.; Alves, O. L.; Fávaro, W. J.; J. Phys.: Conf. Ser. 2015, 617, 012019.

9. Zhang, J.; Feng, L.; Tan, X.; Shi, X.; Xu, L.; Liu, Z.; Peng, R.; Part. Part. Syst. Charact. 2013, 30, 794.

10. Durán, M.; Durán, N.; Luzo, A. C. M.; Duarte, A. S. S.; Volpe, B. B.; Ceragioli, H. J.; Andrade, P. F.; Souza, J. G.; Fávaro, W. F.; J. Phys.: Conf. Ser. 2017, 838, 012035.

11. Feng, L.; Yang, X.; Shi, X.; Tan, X.; Peng, R.; Wang, J.; Liu, Z.; Small 2013, 9, 1989.

12. Sun, X.; Liu, Z.; Welsher, K.; Robinson, J. T.; Goodwin, A.; Zaric, S.; Dai, H.; Nano Res. 2008, 1, 203.

13. Hou, T.; Zhou, X.; Xing, D.; Biomaterials 2014, 35, 4185.

14. Durán, N.; Makita, Y.; Innocentini, L. H.; Biochem. Biophys. Res. Commun. 1979, 88, 642.

15. Fávaro, W. J.; Nunes, O. S.; Seiva, F. R.; Nunes, I. S.; Woolhiser, L. K.; Durán, N.; Lenaerts, A. J.; Infect. Agents Cancer 2012, $7,14$. 
16. Dias, Q. C.; Nunes, I. S.; Garcia, P. V.; Fávaro, W. J.; Int. Braz. J. Urol. 2016, 42, 942.

17. Steinberg, G. D.; Smith, N. D.; Ryder, K.; Strangman, N. M.; Slater, S. J.; Postgrad Med. 2011, 123, 28.

18. Durán, N.; Dini, A. X. P.; WO 2016065444 A1, 2016.

19. Munhoz, F.; Vecchio, R.; Durán, N.; Dini, A. X. P.; de Jesus, M. B.; PCT/BR2016/050087, 2016.

20. Fávaro, W. F.; de Souza, J. G.; Dini, A. X. P.; Muniz, M. S.; Matsumoto, M. Y.; Ferreira, L. A. B.; de Jesus, M. B.; Durán, N.; PIBR 102017004724 5, 2017.
21. Lima, P. A.; Rampazo, C. A. D.; Costa, A. F.; Rodrigues, T.; Watashi, C. M.; Durán, N.; J. Phys.: Conf. Ser. 2017, 838, 012027.

22. Dong, X.; Wang, W.; Qu, H.; Han, D.; Zheng, J.; Drug Delivery 2016, 23, 1374.

23. Gupta, B.; Poudel, B. K.; Tran, T. H.; Pradhan, R.; Cho, H.-J.; Jeong, J.-H.; Shin, B. S.; Choi, H.-G.; Yong, C. S.; Kim, J. O.; Pharm. Res. 2015, 32, 2912.

Submitted: October 12, 2017 Published online: January 25, 2018 